\title{
Molecular identification and comparative characterization of Fusarium isolates, obtained from potato plants
}

\author{
Zarina Kostennikova ${ }^{1, *}$, Yaw Akosah ${ }^{1}$, and Ayslu Mardanova ${ }^{1}$ \\ ${ }^{1}$ Kazan Federal University, Institute of Fundamental Biology and Medicine, 420008, 18 \\ Kremlevskaya st. Kazan, Russia
}

\begin{abstract}
The paper analyzes fungi of the genus Fusarium isolated from plants and tubers of different potato cultivars grown on the territory of Tatarstan. It was established that the majority of the strains belonged to the Fusarium oxysporum species complex. Isolates obtained from the root necks of wilting potato plants and conditionally healthy tubers are, to varying degrees, capable of causing dry rot of tubers during artificial inoculation. Also, the virulence of the strains does not correlate with extracellular proteolytic activity. It was shown that the tubers of the Zhukovskij rannij cultivar are most sensitive to fusaria infections, in contrast to the Red Scarlet and Reggi cultivars.
\end{abstract}

\section{Introduction}

The genus Fusarium has attracted close attention due to their wide distribution in various soils, pathogenicity in relation to important crops, and their role as toxin producers [1]. Fusarium wilt (FW) of potato is a global disease that reduces the yield and quality of crops under conditions of agro-industrial production [2]. In the last decade, there has been an increase in the prevalence of the disease in the Tatarstan region of the Russian Federation [3], and this has raised interest in the identification and characterization of Fusarium isolates associated with potato plants. Despite the large amount of research devoted to the genus Fusarium, information on the species composition and virulence factors of the strains prevalent in certain regions is scarce or fragmentary. Notwithstanding, the damage caused by these fungi is extremely large. The current study aimed to isolate fungi of the genus Fusarium from potato plants of different varieties, characterize their species composition, and assess their pathogenic properties.

\section{Methods of research}

Potato plants of the cultivars Gala, Vendi, Vega, Zhukovskij rannij, Zhuravinka, and Red Scarlet were cultivated in the experimental fields of the Tatar Research Institute of

\footnotetext{
* Corresponding author: tosheva_zarina@mail.ru
} 
Agriculture (Republic of Tatarstan, Laishevsky district). Fungi were isolated from the root necks of plants of these cultivars with signs of FW or conditionally healthy tubers (potato tubers without signs of Fusarium dry rot [FDR]).

The root necks of the stems of potato plants were washed with water from the soil, dried with subsequent removal of the periderm. Using a sterilized scalpel, the stem was cut into pieces of $2-3 \mathrm{~cm}$ in length, which were surface sterilized in a $0.5 \%$ sodium hypochlorite solution for $3 \mathrm{~min}$, then washed 3 times in sterile distilled water, dried, and transferred onto Petri dishes with sterile Potato Dextrose Agar (PDA) [4]. Plates were incubated for 7 days at room temperature. Following the observance of mycelia growth on the tissues, the fungi were transferred onto sterile Czapek-Dox Agar (CDA) [4] or PDA media. To obtain isolates from tubers, the tubers were washed and surface treated with $70 \%$ alcohol and $1 \%$ sodium hypochlorite solution, and the area adjacent to the stolon of the tuber was peeled off with a sterilized scalpel. A disc of 3-4 mm thick was cut and removed. Then 3 discs with a thickness of 2-3 mm were sliced, disinfected for 2 minutes in $70 \%$ ethanol, washed thrice with sterile distilled water, and dried on sterile filter papers. After this, the discs were transferred onto Petri dishes with sterile CDA medium and incubated for 7 days at room temperature.

To obtain pure fungal cultures, the developed colonies with Fusarium-like morphological appearance were reseeded. The obtained isolates were monitored by microscopy for the absence of extraneous microbes. Fungal cultures were grown for 7 days at $28-29^{\circ} \mathrm{C}$ and then stored at $4^{\circ} \mathrm{C}$.

The pathogenicity of the isolates was assessed by their ability to cause FDR when artificially inoculated into tubers with fungal spores. Using a micro-syringe, $20 \mu \mathrm{L}$ of fungal spore suspension $\left(10^{5} / \mathrm{ml}\right)$ was inoculated into each of 10 tubers of the cultivars Zhukovskij Rannij, Red Scarlet, and Reggi [5]. Infected tubers were incubated at $28{ }^{\circ} \mathrm{C}$ for 7 days, then at room temperature for 14 days. Tubers were examined daily during the 21 days of incubation for signs of FDR. After 21 days, the tubers were cut into four lobes along the wounded section for FDR assessment. The presence of diseased tissue in all parts was noted. The virulence of an isolate was determined by the area coverage of FDR, as well as the minimum number of days of incubation until the first signs of FDR. The degree of tuber damage was assessed using the following scale: "." no damage, "+" - the damage affected only one lobe of the tuber, and "++", "+++" and "++++" representing damage observance in 2, 3 and all 4 lobes of the tuber, respectively.

The resistance of tubers to FDR was calculated on a 5-point scale as an inverse relationship to the degree of tuber damage. Tubers without signs of damage were evaluated at 5 points of resistance; 4 points - tubers with damage to only a portion of a tuber with an injection site; Tubers with damage from 2 to 4 tuber lobes, respectively, were assessed from 3 to 1 point of resistance.

Cultivar resistance indices (CRI) to fungal isolates were calculated for all 3 cultivars using the following formula:

$$
\mathrm{CRI}=\left(\sum \mathrm{T}_{\mathrm{H}}-\sum \mathrm{T}_{\mathrm{A}}\right) / \sum \mathrm{T}_{\mathrm{H}}
$$

where TH is the sum of scores of the unaffected tubers and TA is the sum of the scores for all diseased tubers.

The cultivar was considered resistant at $1 \geq \mathrm{CRI}>0$ and susceptible at $0>\mathrm{CRI} \geq-1$. Qualitatively, proteolytic activity was determined by plating cultures on an agar medium containing $10 \%$ skim milk powder, with subsequent incubation at $28{ }^{\circ} \mathrm{C}$ for 3 days, and the presence of proteolytic activity was assessed by the formation of clear zones around the colonies. 
The extracellular proteolytic activity was assayed by the hydrolysis of azocasein (Sigma, United States) according to the method described in [6]. To obtain samples of the crude enzyme (CE), fungi were cultured at a $28 \pm 2{ }^{\circ} \mathrm{C}$. for 7 days in Czapek-Dox broth with aeration at $200 \mathrm{rpm}$ on a shaker-incubator (INFORS HT Standard, Switzerland). Next, the cultures were centrifuged at $3000 \mathrm{rpm}$ at a temperature of $4{ }^{\circ} \mathrm{C}$ for $7 \mathrm{~min}$. A cell-free supernatant containing the $\mathrm{CE}$ was taken and kept frozen at $-20{ }^{\circ} \mathrm{C}$ until the proteolytic activity was determined. A unit of enzyme activity was defined as the amount of CE that hydrolyzes $1 \mu \mathrm{g}$ of the substrate per minute under the experimental conditions.

The species identification of fungal isolates was carried out based on morphological characteristics and molecular analysis. The presence, structure, and abundance of fungal microstructures were analyzed using light microscopy (XSZ-148E Microscope, Zenith lab inc., USA). The DNA of the isolates was isolated by the phenol-chloroform method and used for the amplification of the 5.8S rRNA gene: conserved regions (internal transcribed spacer) using the primers ITS1 (TCCGTAGGTGAACCTGCGG), ITS4 (TCCTCCGCTTATTGATATGC), and the 18S rDNA variable regions using the primers 18S-F (ATTGGAGGGCAAGTCTGGTG), 18S-R (CCGATCCCTAGTCGGCATAG). The sequence data were annotated using the BLAST bioinformatics platform.

Statistical processing of data

Statistical processing of the experimental data was carried out by determining the arithmetic mean values and their standard errors in Microsoft Excel (2019).

\section{Research results and discussion}

\subsection{Fungal isolation}

In total, fifteen fungal isolates were obtained, 7 of which were successfully acquired in pure cultures. All these 7 isolates had conidial characteristics that aligned with the morphology of the genus Fusarium and thus, were used for further investigations. Four isolates were isolated from the root necks of potato plants of cultivars Gala (strain sGaO103), Vendi (sVnO104), and Vega (sVgO108, sVgO110). One isolate each was obtained from the tubers of potato cultivars Zhukovskij rannij (strain tJuO120), Zhuravinka (tJrO112), and Red Scarlet (tRsO117).

\subsection{Molecular identification}

Species identification of the isolates was carried out on the basis of 98-100\% homology of the sequenced amplicons of the ITS and 18S regions of the rDNA gene with the strains of the corresponding species from the NCBI database. We established that 6 strains (sGaO103, sVnO104, sVgO108, sVgO110, tJrO112, tRsO117) belong to the Fusarium oxysporum species complex, while the tJuO120 strain was identified as Fusarium solani.

\subsection{Proteolytic activity}

By culturing the isolates on skimmed milk agar, it was shown that all isolates exhibit extracellular proteolytic activity, possibly due to the secretion of alkaline proteinases. It is known that Fusarium extracellular proteinases play a role in pathological processes during plant infection [7, 8]. A comparative analysis of the level of accumulation of Fusarium extracellular proteinase activity on the 7th day of growth in Czapek Dox Broth (CDB) was carried out. As observed in Figure 1, the proteinase activity of the isolates ranges from 1.25-2.27 $\mathrm{U} / \mathrm{ml}$. The maximum production of extracellular alkaline proteinase was 
observed in the $F$. oxysporum $\mathrm{sVnO} 104$ strain, while the minimum activity was registered in the $F$. solani tJuO120 strain.

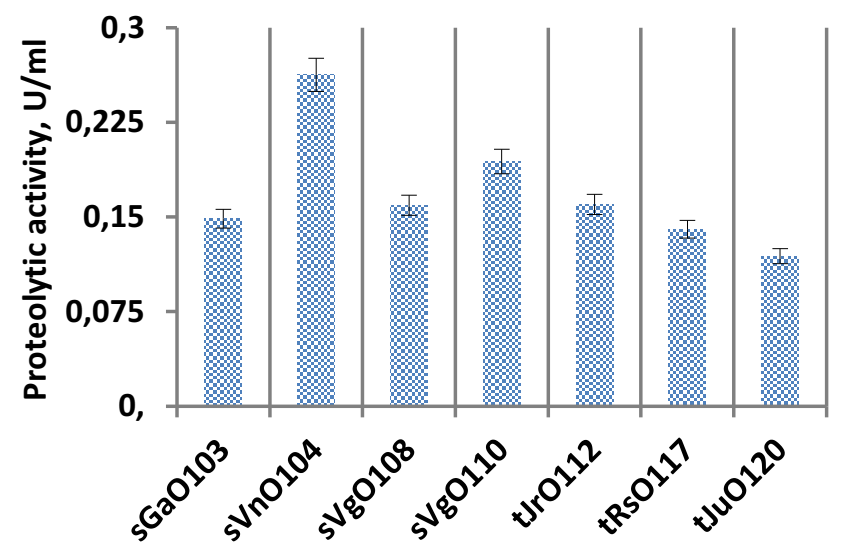

Fusarium isolates

Fig. 1. Proteolytic activity (using azocasein as substrate) of the crude enzymes of Fusarium isolates on the $7^{\text {th }}$ day of growth

\subsection{Pathogenicity}

It is known that Fusarium strains are capable of causing dry rot of potato tubers at varying degrees, which can lead to significant yield losses during storage [9]. Following the artificial inoculation of tubers, it was observed that all the studied Fusarium isolates have the ability to cause FDR at varying degrees, which indicates their virulent properties (table 1). It was also noticed that the virulence and aggressiveness of strains differ in tubers of different potato cultivars.

Table 1. Characterization of the pathogenic properties of Fusarium isolates

\begin{tabular}{|c|c|c|c|c|c|}
\hline \multirow[t]{2}{*}{ isolate } & \multicolumn{2}{|c|}{ Isolation source } & \multicolumn{3}{|c|}{$\begin{array}{l}\text { Virulence of isolate in relation to tubers } \\
\text { of different cultivars: day of onset of the } \\
\text { first FDR symptoms and the degree of } \\
\text { tuber damage* }\end{array}$} \\
\hline & $\begin{array}{l}\text { Vegetative } \\
\text { organ }\end{array}$ & Cultivar & $\begin{array}{c}\text { Zhukovskij } \\
\text { rannij }\end{array}$ & Reggi & $\begin{array}{c}\text { Red } \\
\text { Scarlett }\end{array}$ \\
\hline $\begin{array}{l}\text { F. oxysporum } \\
\text { sGaO103 }\end{array}$ & Root neck & Gala & $14,++$ & $>21,-$ & $17,++$ \\
\hline $\begin{array}{l}\text { F. oxysporum } \\
\text { sVnO104 }\end{array}$ & Root neck & Vendi & $14,++++$ & $17,++$ & $>21,-$ \\
\hline $\begin{array}{l}\text { F. oxysporum } \\
\text { sVgO108 }\end{array}$ & Root neck & Vega & $14,++$ & $17,+$ & $>21,-$ \\
\hline
\end{tabular}




\begin{tabular}{|c|c|c|c|c|c|}
\hline $\begin{array}{c}\text { F. oxysporum } \\
\text { sVgO110 }\end{array}$ & Root neck & Vega & $17,++$ & $>21,-$ & $14,+$ \\
\hline $\begin{array}{c}\text { F. oxysporum } \\
\text { tJrO112 }\end{array}$ & Tuber & Zhuravinka & $11,+++$ & $>21,-$ & $>21,-$ \\
\hline $\begin{array}{c}\text { F. oxysporum } \\
\text { tRsO117 }\end{array}$ & Tuber & Red Scarlett & $8,+++$ & $>21,-$ & $14,++$ \\
\hline F. solani tJuO120 & Tuber & $\begin{array}{c}\text { Zhukovskij } \\
\text { rannij }\end{array}$ & $4,+++$ & $8,++$ & $14,+++$ \\
\hline
\end{tabular}

* «-» - the absence of tuber damage; «+» - dry rot affects one lobe of a tuber, «++», «+++», and «++++» - lesions observed in 2, 3, or 4 lobes of a potato tuber, respectively.

As seen from the table, all strains exhibit different levels of virulence against potato tubers, depending on the tested cultivar. It was found that $F$. solani tJuO120 exhibits high virulent properties, causing dry rot in tubers of all three varieties, and in the case of Zhukovskij rannij, lesions begin to develop as early as 4 days after inoculation. The $F$. oxysporum sVnO104 strain showed high aggressiveness towards the tubers of Zhukovskij rannij, causing dry rot damage to the entire tuber.

A comparative analysis of the degree of infestation of tubers of different varieties showed that tubers of the Zhukovskij rannij $\left(C_{R I}=-0.1\right)$ were the most sensitive to dry rot damage, while the Reggi $\left(\mathrm{C}_{\mathrm{RI}}=0.86\right)$ and Red Scarlett $\left(\mathrm{C}_{\mathrm{RI}}=0.64\right)$ showed relatively high resistance to isolates.

\section{Conclusion}

Pure cultures of 7 Fusarium isolates were isolated from conditionally healthy tubers and the root necks of potato plants with signs of FW. One of the isolates was identified as F. solani, while the remaining six belonged to the $F$. oxysporum species complex. All isolates exhibited extracellular proteolytic activity due to the action of alkaline proteinases. The $F$. oxysporum $\mathrm{sVnO} 104$ strain isolated from the root neck of the Vendi cultivar exhibited the maximum proteolytic activity. However, during the artificial inoculation of tubers, the ability to quickly induce dry rot in all three varieties was exclusively demonstrated by the strain $F$. solani tJuO120. It was found that the tubers of different potato cultivars differ in resistance to $\mathrm{FW}$, and the most sensitive were the potato tubers of the Zhukovskij rannij cultivar.

\section{Acknowledgment}

This work was funded by a grant from the Russian Foundation for Basic Research (Project no. 19-316-90028).

\section{References}

1. T.Y. Gagkaeva, O.P. Gavrilova, M.M. Levitin, MYCOLOGY AND PHYTOPATHOLOGY, 42(3), 201-214 (2008)

2. R. Dean, Z.A. Pretorius, K.E. Hammond-Kosack, A.Di Pietro, P.D. Spanu, G.D. Foster, Mol. Plant Pathol., 13(4), 414-430 (2012) 
3. F.F. Zamalieva, T.W. Zaizeva, L.Y. Rygih, Z. Z. Salikhova, Zashchita kartofelia, 2, 39 (2015)

4. V.D. Poliksenova, A.K. Khramtsov, S.G. Piskun, Mycology. Methods for the experimental study of microscopic fungi, 1-42 (2012)

5. E. Stefanczyk, S. Sobkowiak, M. Brylinska, J. Sliwka, Eur. J. of Plant Pathol., 145(4), 871-884 (2016)

6. I.V. Demidyuk, D.V. Romanova, I.V. Nosovskaya, E.A. Demidyuk, G.G. Chestukhina, I.P. Kuranova, S.V. Kostrov, Protein Eng. Des. Sel., 17(5), 411-416 (2004)

7. M.J. Nightingale, B.A. Marchylo, J.E. Dexter, K.R. Preston, Cereal Chem., 76(1), 150158 (1999)

8. F. Olivieri, M.E. Zanetti, C.R. Oliva, A.A. Covarrubias, C.A. Casalongué, Eur. J. of Plant Pathol., 108(1) ,63-72 (2002)

9. G.F. Hadieva, M.T. Lutfullin, Y.A. Akosah, A.V. Malova, N.K. Mochalova, S.G. Vologin, A.M. Mardanova, Dostizheniya nauki i tekhniki APK, 32(3) (2018) 